\title{
Reliability of the input admittance of bowed-string instruments measured by the hammer method
}

\author{
Ailin Zhang and Jim Woodhouse \\ Dept. of Engineering, University of Cambridge, Trumpington Street, Cambridge CB2 1PZ, UK ${ }^{\mathrm{a}}$ )
}

(Dated: November 4, 2014)

\begin{abstract}
The input admittance at the bridge, measured by hammer testing, is often regarded as the most useful and convenient measurement of the vibrational behaviour of a bowed string instrument. However, this method has been questioned, due especially to differences between human bowing and hammer impact. The goal of the research presented here is to investigate the reliability and accuracy of this classic hammer method. Experimental studies were carried out on cellos, with three different driving conditions and three different boundary conditions. Results suggest that there is nothing fundamentally different about the hammer method, compared to other kinds of excitation. The third series of experiments offers an opportunity to explore the difference between the input admittance measuring from one bridge corner to another and that of single strings. The classic measurement is found to give a reasonable approximation to that of all four strings. Some possible differences between the hammer method and normal bowing and implications of the acoustical results are also discussed.
\end{abstract}

PACS numbers: 43.75.De

\section{INTRODUCTION}

In the context of the acoustics of bowed string instruments, the input admittance (or point mobility) is normally defined as the ratio of the mechanical velocity of the attachment point of the string on the bridge to the driving force applied at the same point (Jansson, 1999). It is commonly used to capture the essential acoustical properties of the instrument body. It is also a good choice to relate the vibration characteristics to questions of sound quality and playability of the instrument, for several reasons.

Firstly, for all stringed instruments, the string is the source of energy and the bridge is the main connection point to the body. There is always a relatively weak coupling between the strings and the instrument body because of their significant impedance difference at the point of contact. This impedance mismatch leads to the notion that the input admittance contains the major information about the energy transfer from string to body, although it gives no direct information related to the radiation of sound (Cremer, 1985). Second, the measurement of the input admittance is easy to reproduce and calibrate, and can offer significant information about the vibrational characteristics of instruments using a variety of analysis approaches. Third, it is generally assumed that the body vibration is well approximated as a linear dynamic system, but the stick-slip string motion in response to a given bow gesture is strongly nonlinear. Body motion can feed back to influence the response of the string to bowing, and such influences on 'playability' are captured, approximately, by the input admittance at the bridge (Woodhouse, 1993).

Similar to any other mechanical vibration response,

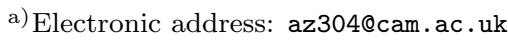

the input admittance of a violin is dominated by discrete modal peaks at lower frequencies, where the modes are well separated, while at higher frequencies the modes begin to overlap and statistical features become significant. The most widely-discussed such statistical feature is the so-called 'bridge hill' (Jansson , 1997). The focus of the present paper is on measurement rather than interpretation of the admittance, so detailed discussion is not given here: see Cremer(Cremer, 1985) for a summary of the early work by Reinicke and others in the Berlin research group, and Woodhouse (Woodhouse, 2014) for a more recent general review of violin body vibration.

The most common measurement method was pioneered by Jansson (Jansson , 1997), and involves measuring the mechanical input admittance using a miniature force hammer tapping one corner of the bridge of the instrument and collecting the motion of the bridge from the other corner. Strictly, any such measurement is a transfer admittance rather than a point admittance because the driving and measuring positions are different. However, provided the separation is not too large the result can give a useful approximation to the point admittance. An instrumented hammer arranged as a pendulum is employed to excite the bridge corner over a frequency range of interest. The response velocity or acceleration signal at the other corner is collected by a laser vibrometer, or by a lightweight accelerometer which is mounted on the bridge with superglue. Acceleration response can be converted to mobility by data analysis software.

For this method, all the strings are thoroughly damped by a piece of paper woven between them: the requirement of adequate damping is that no musical pitch can be heard when the strings are plucked. The purpose of damping is to explore the vibration characteristics of the body without the effect of string resonance. The after-lengths of the strings, between the bridge and the tailpiece, were left undamped. Before any measurement, the strings are tuned to their usual playing tensions so 
that the bridge, body and soundpost are supported and stressed exactly as in normal playing conditions. Also, the influence of the axial stiffness of the strings on the body modes is maintained.

However, doubts have been raised over the reliability and validity of this classical measurement. British violin maker Nigel Harris has suggested that there are certain differences between human bowing and hammer impact that might give rise to problems and even greatly affect the result in his experiments(Harris and Fahy, 2009). This naturally invites the question of whether using the hammer method is significantly different from exciting the violin by playing with a bow in the usual manner. In normal playing, Helmholtz motion (see Cremer(Cremer, 1985) Chapter 3) of the bowed string results in the instrument being excited by means of a sawtooth wave of force at the string notch in the bridge. The vibrating string will also exert force on the instrument body at its other end, on the fingerboard. In hammer testing, the impulse applied at the driving point resembles a half-cycle sinusoidal pulse, with finite time duration. The excitation is at the side of the bridge, a little distance away from all the string notches.

There are other issues that could influence the accuracy and reliability of the hammer method. In the case of a bowed-string instrument, the most important direction of the motion of a vibrating string is the bowing direction, which is approximately parallel to the top plate. This input force given by bowing is converted into forces at the bridge feet perpendicular to the top plate, so as to excite predominantly bending vibration of the belly. Thus the one-dimensional input admittance measuring from corner to corner of the bridge, both in the bowing direction of their nearest strings, can only be an approximation to that of each of the separate strings. Indeed, the input admittance will not be exactly the same for all four strings because each string has a slightly different bowing direction and different coupling point on the bridge.

The fixture used to hold the instrument will also have an influence on the measured behaviour, and might possibly contribute to differences between excitation methods (Marshall and Genter, 1986). The influence from different fixtures on the experimental results will be discussed in later sections.

The main purpose of this paper is to test the accuracy and reliability of the classic hammer method for measuring the bridge admittance of bowed string instruments. Systematic experiments were conducted on a cello, always in the same laboratory environment. There are three sets of measurements. The first set was to investigate the effect of three different forcing methods: controlled input force was applied to a cello separately by an instrumented hammer, a breaking wire, and regular bowing. In the second set of experiments, the holding methods on the cello were varied: the instrument was supported on soft foam while resting on its back on a bench, held in the hands of a player as for normal playing, and held in a frame with supports which approximately mimics a player's hold. An attempt has been made in the third set of experiments to survey input admittances for the four separate cello strings, and compare the results with the classical single measurement. In addition to these three sets of experiments, comparisons between results measured with different added mass and on different dates will be presented, to explore how the frequency response of an instrument is influenced by other factors.

\section{BACKGROUND}

In the classic hammer measurement procedure for the mechanical input admittance(Fritz et al., 2007), an instrument is driven by means of a miniature impulse hammer at one corner of the bridge in the bowing direction of the nearest string while the resulting motion of the bridge is measured on the other top corner of the bridge, in the bowing direction of the nearest string, by a laser vibrometer or small accelerometer. The input admittance can be found by Fourier analysis of the force and velocity signals, after the measured acceleration signal has been converted to the response velocity. In the frequency domain, the driving force $F(\omega)$ and the response velocity $V(\omega)$ are related by a scalar transfer function $Y(\omega)$ :

$$
V(\omega)=Y(\omega) F(\omega)
$$

This equation relies on the assumption that the whole experimental chain is a linear system, so the amplitude and waveform of the input signal should not affect the input admittance.

The gradual development of experimental methods for this task is documented in the literature. Early measurements were made in the 1970s in Cremer's research group (see Cremer(Cremer, 1985) Chapter 10). At about the same time, a technique for measuring one-dimensional input admittance was developed in the laboratory of Jansson. The simple driving system Jansson presented, consisting of an impedance head and strong magnets, was improved further by Firth (Firth , 1976) and was then widely applied to the measurement of many instruments. Jansson (Jansson , 1997) later increased the range of this measurement up to $10 \mathrm{kHz}$ by replacing the impedance head with a magnet-accelerometer system. In this system, a magnet and miniature accelerometer were fastened to the instrument bridge. Force was applied via an electrical coil held close to the magnet, driven with constant current so as to produce a constant driving force. The output signal was collected from the accelerometer. Taking advantage of this system, Jansson also mapped out several eigenmodes of different stringed instruments (?). In an alternative approach, Trott (Trott, 1987) used a rod linking a shaker to the violin bridge. The first study of acoustical properties of the violin performed with an impact hammer was made by Jansson, Bork and Meyer in 1986 (Jansson, 1986). They showed that impulse response measurements can produce fast and sufficiently detailed mechanical admittance. Since then the hammer method has been applied widely in musical instrument research.

In addition to certain earlier optical investigations, Jansson and Molin(Jansson, 2006) introduced the Laser 
Doppler Vibrometer system to measure the vibration of the violin instead of the accelerometer. By combining it with the impulse hammer, the input admittance can be measured without any extra mass or damping being added to the instrument.

However, all these measurements only deal with the mobility relating one direction of force and one direction of motion. In practice, it can be hard to keep the force in a single well-controlled direction or at a single mechanical point, due to the limitations of the experimental setup. In any case, the true input force from a bowed string is not confined precisely to a single plane: the player can vary the bowing angle somewhat, especially on the outer strings. Idealization of the input force as lying in a single plane might therefore be inappropriate: in general it might be necessary to consider two or even all three components of the input force and of the resulting velocity, requiring a matrix of transfer functions.

Lambourg and Chaigne (Lambourg, 1994) aimed at measurements of a $2 \times 2$ admittance matrix, which deals with two directions for both force and response. However, this method was still subject to some problems according to Boutillon et.al. Boutillon and Weinreich (Boutillon and Weinreich, 1988) presented the theoretical background of a 3-dimensional admittance matrix and proposed a new method to realize the measurement. Three ultralight accelerometers were used to pick up vibration of the bridge by comparing the motion under different external loading conditions. Further investigation was reported by Yoder (Yoder, 1991) using a one-dimensional mass-loading technique, which decomposed the three-dimensional motions to three independent problems. Some direct measurements of the $3 \times 3$ admittance matrix on a cello were reported by Woodhouse and Courtney (Woodhouse and Courtney, 2003), using a specially modified bridge to allow the hammer and laser vibrometer to be used in all three directions near a single point.

\section{EXPERIMENTAL PROCEDURES}

\section{A. Experimental Setup}

The experiments reported here were carried out on a cello of moderate quality, all in the same laboratory acoustic environment. There is no special requirement on the acoustical field of the room because sound radiation and reverberation makes a negligible difference to measurements of input admittance provided the acoustical environment is reasonably normal: for example a lowloss reverberation chamber might reduce modal damping factors of the instrument body.

A small accelerometer (DJB M2222C, DJB Instruments, Suffolk, UK) glued on the C-string corner of the cello bridge was used for the measurement of vibration response. Adhesive mounting allowed the accelerometer to be easily removed from the bridge without damaging anything. Smooth and flat contacting surfaces between the bridge and the accelerometer are preferred for obtaining the best accuracy and high-frequency transmissibility.
In addition, the accelerometer was properly orientated so that its central line was parallel to the primary vibration direction of the cello $\mathrm{C}$ string, namely the bowing direction.

The main disadvantage of an accelerometer lies in the fact that the extra mass contributed by it and its connecting cables will modify the vibration response of the instrument. Thus the accelerometer $(0.5 \mathrm{~g})$ used in our experiments represents a compromise in the interests of practicality.

It would in principle be better to use a laser vibrometer rather than an accelerometer. A considerable advantage of the laser set-up is that no added mass is involved. It can also detect the response signal in any position on the instrument under test. However, the laser approach is not feasible for the present purpose since its signal stability is very sensitive to the precise alignment and reflection quality of the laser beam. It is easy enough to use the laser for tests using the impulse hammer for force input, but the other forcing approaches used in this paper pose problems. Particularly for excitation by bowing, it is very hard to maintain a good laser signal in the presence of disturbances a player inevitably imposes on the cello body during bowing and fingering the strings. It was judged better for clean comparisons within the main sets of experiments to be reported here to use an accelerometer throughout.

In order to minimize the mass-loading effect of the accelerometer, all experiments in this paper were undertaken on a cello rather than a violin. The heavier bridge of the cello reduces the relative influence of the accelerometer mass because the size and weight of a cello bridge are considerably larger than those of a violin. A typical example of the mass-loading problem is presented in Figure 1: the plot emphasises the difference between input admittances of a violin using the accelerometer and a laser vibrometer (Polytec OFV-056, Polytec, Harpenden Hertfordshire, UK). The result measured by the accelerometer (indicated by the dashed line) drops dramatically in amplitude at higher frequencies compared with that measured by the laser vibrometer (indicated by the solid line). This downward trend caused by the extra mass completely eliminates the bridge hill, the broad peak of response around $2.5 \mathrm{kHz}$ in the admittance of the violin under test. All figures in this paper show calibrated admittance magnitude in $\mathrm{dB}$ re $1 \mathrm{~ms}^{-1} \mathrm{~N}^{-1}$.

For a cello, the accelerometer and laser vibrometer yield nearly identical results as shown in Figure 2. The bridge hill is clearly visible in both results for the cello under test, in the vicinity of $1 \mathrm{kHz}$. All comparisons between different driving and boundary conditions in this paper are illustrated by results from this cello.

Even so, the ability of the cello to tolerate added mass loading is very limited. The input admittance of the cello is sensitive to extra mass not only from the accelerometer but also from the force-measuring sensors mounted on the bridge as a necessary part of these particular measurements. This will give rise to some complications in the measurements and their interpretation, as will be explained in detail below and illustrated with measurements in Sec. IV A. 


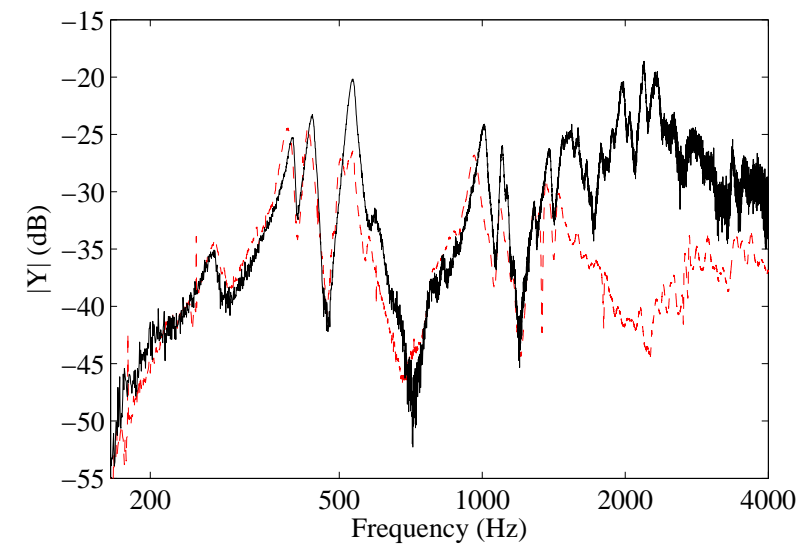

FIG. 1. Bridge input admittance for the tested violin measured by an accelerometer (dashed line) and a laser vibrometer (solid line).

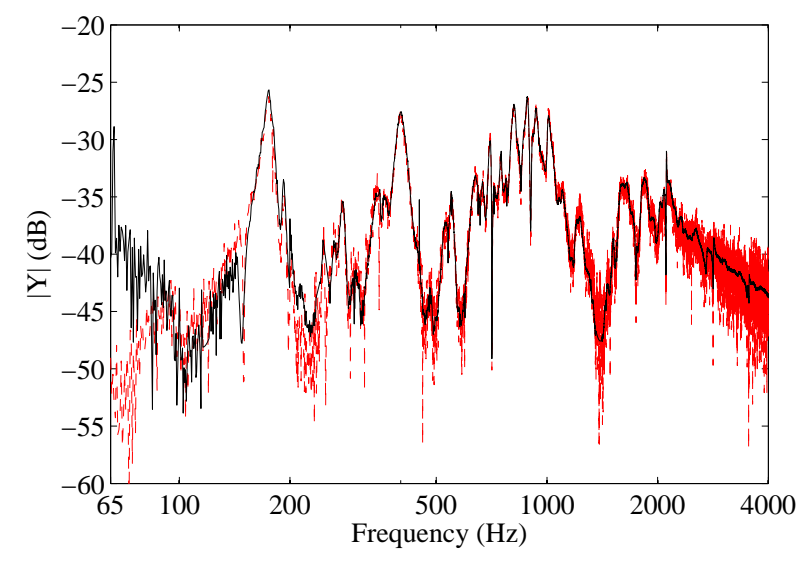

FIG. 2. Bridge input admittance for the tested cello measured by an accelerometer (solid line) and a laser vibrometer (dashed line).

For collecting the input force signals, two approaches were used. In the first approach, a miniature force hammer (PCB 086D80, PCB Piezotronics, NY, USA) fitted with a piezoelectric force transducer was employed to excite the cello bridge at the A-string corner. However, one cannot be sure of the precise direction and location of the impact force, since the steel tip of the hammer is hitting relatively soft wood with local inhomogeneity from the grain structure. To address this problem, the hammer was attached to a pendulum fixture so that it is easier to ensure a consistent impact, perpendicular to the surface at the same point on the bridge every time. Another problem arises from the limitation of the frequency range. The input energy in the high frequency range may be very low, making the results at higher frequencies noisy and unreliable. A hard steel impact tip was preferred since it can provide the broadest frequency range. Calibration checks were made on the hammer measurements using a standard technique based on a ballistic mass (see for example(Ewins, 1986)).

An alternative approach was to measure the force ex- erted at a particular string notch on the bridge, using a bridge pick-up system. This pick-up system consists of two small pieces of piezoelectric crystal mounted beneath the required string notch on the bridge in a Vconfiguration. The crystals produce two voltages that can be combined to give a signal proportional to the transverse force at the string notch in the bowing direction. These embedded sensors do not interfere with the transverse motion of the string, so they can be used with normal bowing. The same sensors also work for the wirebreak excitation method, described shortly. For the final experiment, involving measurements for all four separate strings, four such sensors were needed.

Both the input and output signals were fed through charge amplifiers, which were adjusted to appropriate gain levels. Signals were converted at a $40 \mathrm{kHz}$ sampling rate by a National Instruments acquisition card (type 6250) controlled by data logging software written in MATLAB. Averaged transfer functions were finally obtained using a computer. One practical requirement from Eq. (1) is that the linearity of the whole chain of experimental set-up should be checked: this is conveniently done via the associated coherence function.

\section{B. Experiments with different driving methods}

In the first set of experiments, the cello was laid on a table and supported by soft foam pieces during measurements. Three different driving conditions were compared: hammer, step excitation by a breaking wire, and normal bowing of a string. Sample results will be illustrated in Sec. IV B.

Measurements by the hammer method will be described in terms of coordinate systems based on the two bridge corners as shown in Figure 3: the axes X and X' are parallel to the bowing directions of the A string and $\mathrm{C}$ string in the bridge plane; the axes $\mathrm{Y}$ and $\mathrm{Y}^{\prime}$ indicate the directions perpendicular to bowing in the same plane. As already described, impulses are carefully applied to the driving point along X; similarly the output signal collected by the accelerometer at the other side of the bridge is determined along the direction X'. The response along the $\mathrm{Y}^{\prime}$ direction is ignored since the accelerometer used here is a single-axis one. To obtain the best signal-to-noise ratio, it is necessary to generate multiple measurements and compute an average value.

For both the wire-breaking and bowing methods, the measurements used the bridge pick-up system to measure the input force. In the wire-breaking method, a loop of fine copper wire with a diameter of $0.1 \mathrm{~mm}$ was put around the $\mathrm{C}$ string, up against the face of the bridge. The breaking strength of the copper wire is around 1.5 $\mathrm{N}$. The loop can be gently pulled in the desired direction until it breaks, to impart a step function of excitation to the string at the contact point with the bridge. The input force signal was collected by the sensors described above. The measurement was taken with all strings damped. The measurement is easily reproducible using this method since some of the problems of hammer impact have been tackled. Furthermore, due to the 


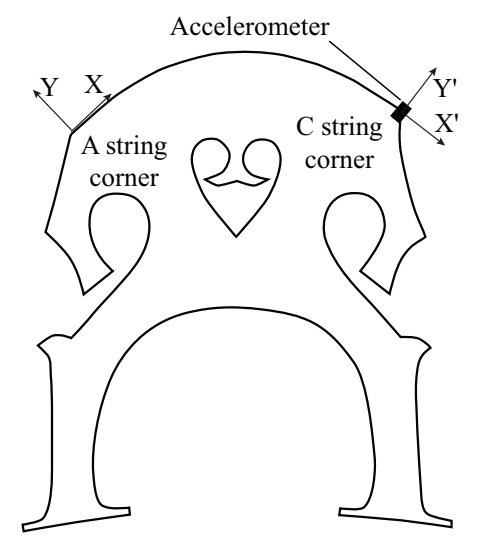

FIG. 3. Coordinate systems used for hammer method.

well-controlled direction and location of force generated by the wire-breaking, this method can be applied to investigate the exact effective angles of the force sensors used for measuring the input signals.

Figure 4 shows the coordinate systems used with this method. The axes X1, Y1, A1, and B1 are consistent throughout the wire-breaking and bowing methods. Based on the $\mathrm{C}$ string notch, the bowing direction of the $\mathrm{C}$ string in the plane of the bridge is denoted $\mathrm{X} 1$, and $\mathrm{Y} 1$ is perpendicular to $\mathrm{X} 1$ in the same plane. A1 and $\mathrm{B} 1$ indicate the directions of input force measured by the two force sensors: they are at approximately 45 degree to the X1 direction, but the exact angles are determined by the calibration procedure.

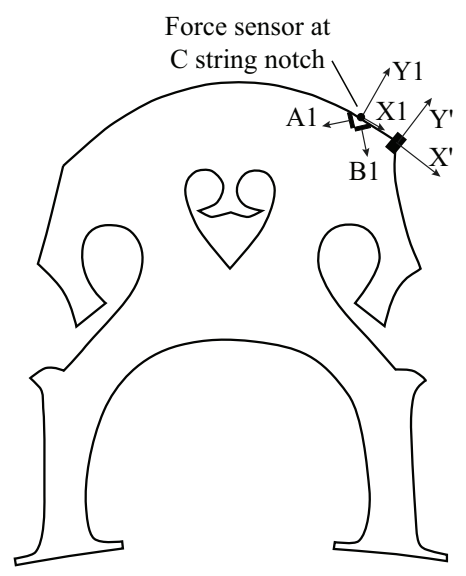

FIG. 4. Coordinate systems used for both wire-breaking and bowing method.

During the calibration, wire-breaks were applied at a range of angles from 0 degree to 90 degree, easily measured and controlled by means of a small protractor mounted to align with the X1-Y1 coordinates. The actual breaking force of the copper wire can be determined by tests using suspended weights to break the wire: the breaking force for copper is very consistent for different samples of wire from the same reel. By fitting calibration factors to these measurements, the input force can be resolved accurately to the required direction and also the magnitude of transfer functions can be converted into physical units.

Unfortunately the wire-break method gives relatively low energy input, and so is prone to noise pick-up, resulting in inaccurate measurements. The effect of noise can be reduced, to a certain degree, by averaging measurements. Another problem is the fiddliness of the wirebreaking, which makes the method much more timeconsuming than the hammer method.

The third approach is the bowing method: bowing on the $\mathrm{C}$ string can be used to generate response curves under rather natural conditions. Some previous work has been done by bowing a single note to measure the input admittance (Harris and Fahy, 2009), but this only provides input force at individual frequencies at the harmonics of the played note. Instead, in the measurements presented here a one-octave glissando was playing on the cello $\mathrm{C}$ string (by the player sliding a finger along the string while bowing). By covering at least an octave, the entire frequency range is filled in: energy is supplied by one or more harmonics at some stage. A sonogram of the measured force from the bridge pick-up during such a glissando is shown in Figure 5. It shows the fundamental frequency sweeping upwards over the played range, and a rich set of harmonics tracking in proportion. During the bowing, the $\mathrm{C}$ string was undamped but the other strings were still thoroughly damped. The bowing parameters were controlled to maintain Helmholtz motion in the $\mathrm{C}$ string, but there is some advantage to using rather scratchy bowing since that helps further to fill in any frequency gaps. The experimental set-up and coordinates were the same as for the wire-breaking method. The component of force at the bridge in the direction X1 is measured by the force sensors, just as in the wire-break method, and used as the input signal in the calculation of the admittance.

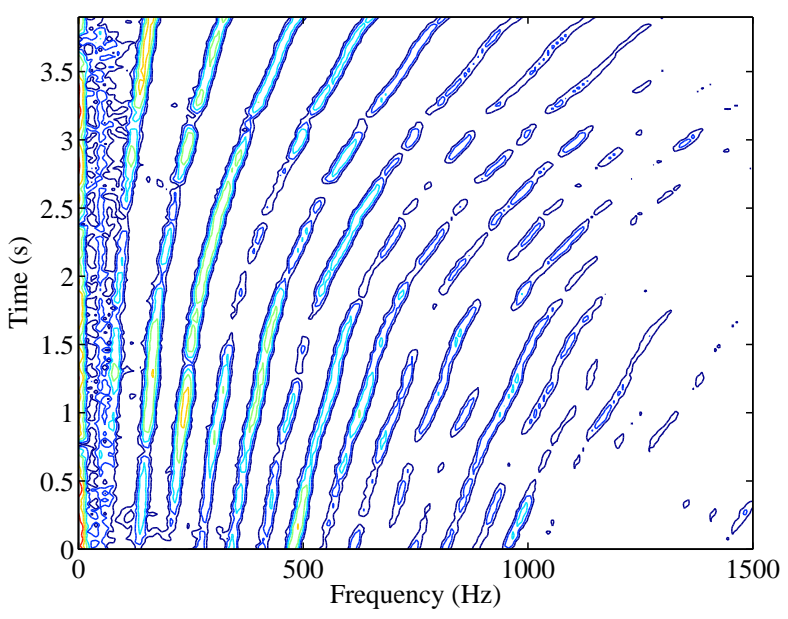

FIG. 5. Measured sonogram of a glissando on the C string of a cello.

By bowing, there is no doubt that the right kind of force is applied to the body, located in the right place. Although such bowing from a player is the closest to the normal playing condition that can deliver credible 
measurements, there are some intrinsic disadvantages: Firstly, the instrument needs to be held firmly enough to bow. But a firm supporting fixture like a clamping arrangement might change the vibration properties of the instrument. However, the mode frequencies are little affected by supporting the cello on its back on blocks of soft foam, and this support method was used here and found to be firm enough for careful bowing. Furthermore, it is likely that not all components of either force or vibration excited by bowing will be detected by the sensors since only one axis of force input and acceleration output were recorded.

\section{Experiments with different holding methods}

To investigate how different boundary conditions affect the measured input admittance, three different holding configurations were compared. Experimental results will be given in Sec. IV C. As mentioned above, the cello was initially set on a flat table, supported on its back by a layer of soft foam. The foam is of moderate thickness with one side being flat and the other side wavy, further reducing the effective stiffness. The foam inevitably provides some additional damping: quantitative results will be shown later. The main advantage with this support method is that it approximates the free-free condition: any additional resonances are at very low frequency and do not interfere significantly with the desired vibration modes of the instrument. It is also the simplest set-up.

To evaluate the potential influence from different holding methods, two alternative methods were also used. For all these tests hammer excitation was used. The input force was applied to the A string corner of the cello bridge and the accelerometer was used to measure the response at the $\mathrm{C}$ string corner. The data processing of input and response signals remained the same as the previous experiment.

When investigating the vibrational behaviour of any musical instrument, holding by a player is a natural choice. For these measurements, the player sat on a chair and held the cello in the normal way, with the endpin resting on a firm floor and the player holding the neck in first position and gripping the body lightly between the knees. It required some care to ensure that the hammer impulse was provided in the bowing direction and in a way consistent in detail with the other tests.

The third holding method was intended to mimic the player's hold. The cello was placed within a steel support frame with a firm base, and steadied by soft foam pieces from two sides in a similar way to the player's knees. Its endpin was located in a hole at the base of the rig, and the neck was fastened by a cable tie to a shaped and rubber-lined block behind the neck in first position. This support method is attractive because it is safe and accurately repeatable while closely resembling the holding manner of a cellist. This made it easy to ensure that the input force and response motion were collected from the bowing directions on the nearby strings during admittance measurements.

\section{Experiments for the separate strings}

The previous two investigations were made with one question in mind: whether the classical single measurement of input admittance from one bridge corner to the other gives a sufficiently good approximation to the separate response at each string notch. To seek the answer to this important question, four separate measurements of transfer function of each string were carried out on the cello. Four sets of bridge pick-up system, as described above, were embedded under the four string notches separately on the cello bridge. One string at a time was excited by wire breaking or bowing, and the transverse input force signal collected from the relevant sensor. The vibration response was measured by the same accelerometer, mounted on the $\mathrm{C}$ string corner of the cello bridge. Measurements were made with all strings damped for comparison with the standard hammer testing while using the wire-breaking excitation. During bowing, the string under test was undamped while the other strings were still thoroughly damped by a piece of paper or the finger of the player. All the bowing used glissando throughout. The signals were processed as before to yield the input admittance for separate strings, shown in Sec. IV D.

\section{SAMPLE RESULTS}

All the input admittances to be shown in this section are calibrated, and are plotted on a decibel scale relative to $1 \mathrm{~ms}^{-1} \mathrm{~N}^{-1}$. The transfer functions were obtained by repeating each measurement at least 15 times without interruption. A frequency range of $65-4000 \mathrm{~Hz}$ is shown, starting from the lowest played note of the cello and covering most of the interesting dynamic response of the body. The coherences of these results were all verified to be close to unity over this frequency range, which suggests that the relation between the input and output is indeed linear to good accuracy. For clarity of discussion, the input admittances are grouped in a sequence of two-way comparisons.

\section{A. Repeatability and the influence of force sensors}

Before looking at the results of the three sets of experiments described above, it is necessary to investigate further the influence on the frequency responses of added mass mounted on the cello bridge, beyond the effect of the accelerometer mass that has already been discussed. The reason is that the various measurements to be shown were taken at different times, and required different numbers of force-measuring sensors fitted to the bridge. These sensors, with their cables, contribute additional mass which has an effect on the results, as will be shown shortly. Each pairwise comparison to be shown was obtained with the bridge in an identical state, but the reader needs to be aware that comparisons between different plots sometimes show the effects of the additional sensors. 
The input admittances of the test cello when the bridge was equipped with one pick-up and three pick-ups are shown in Figure 6. Strong similarity can be seen near the resonance peak at about $172 \mathrm{~Hz}$ (probably the B1mode), which corresponds to the wolf note of the cello under test. Somewhat unexpectedly, the addition of extra sensors results in a slight decrease of the damping factor of this wolf note resonance: measured modal parameters for this mode are given in Table I. At higher frequencies the trends of the two plots are generally similar, but there are obvious and significant differences of detail, especially around $1 \mathrm{kHz}$ where the average amplitude level of the dashed curve is lower than that of solid one.

A similar comparison between the cases of three and four force pick-ups is shown in Figure 7 . The dashed curve in the plot again denotes the results obtained with three pick-ups on the bridge, while the solid one indicates the results with four. Further reduction of amplitude at higher frequencies has occurred with the addition of the fourth pick-up. It is interesting to compare the three results from Figures 6 and 7 . This reveals that the bridge hill in the input admittance curve is erased gradually as the extra mass on the cello bridge increases.

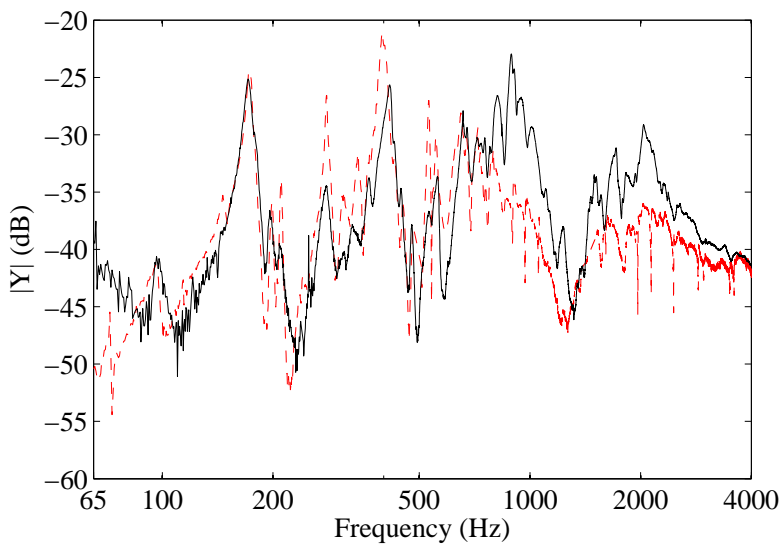

FIG. 6. Bridge input admittances for the tested cello with one force pick-up (solid line) and three pick-ups (dashed line).

TABLE I. Measured resonance frequencies, damping factors and amplitude level for the largest low-frequency resonance.

\begin{tabular}{|c|c|c|c|}
\hline $\begin{array}{c}\text { Sets of sensors } \\
\text { mounted on the } \\
\text { cello bridge }\end{array}$ & $\begin{array}{c}\text { Resonance } \\
\text { frequency } \\
(\mathrm{Hz})\end{array}$ & $\begin{array}{c}\mathrm{Q} \\
\text { factor }\end{array}$ & $\begin{array}{c}\text { Amplitude } \\
\text { level }(\mathrm{dB})\end{array}$ \\
\hline 1 & 171.7 & 18.8 & -25.2 \\
\hline 3 & 173.3 & 26.2 & -24.4 \\
\hline 4 & 173.9 & 24.8 & -25.4 \\
\hline
\end{tabular}

The minimum added mass to allow useful measurements to be made by the three methods described in Sec. III B requires one bridge pick-up plus the attached accelerometer. All the measurements to be shown in Sec. IV $\mathrm{B}$ and $\mathrm{C}$ were done this way, to show the cello in a condition as close as possible to its normal playing state.

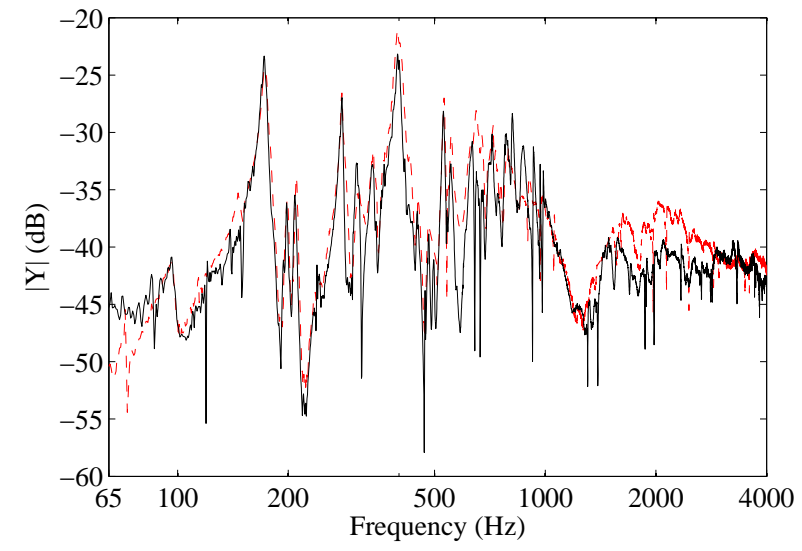

FIG. 7. Bridge input admittances for the tested cello with three pick-ups (dashed line) and four pick-ups (solid line).

However, from the results shown here it is clearly not possible to obtain input admittance with a normal bridge hill shape when the added mass on the cello bridge is greater: essentially, the cello is then being measured with a mute in position. This mass-loading effect will be seen again later, when four pick-ups embedded in the cello bridge were necessary in order to measure the individual velocity responses at the four string notches. For this set of tests only, a different bridge was used with the four pickups.

Before proceeding to the comparison of different driving methods, it is useful to have an indication of the level of repeatability that can be expected from these measurements. Figure 8 shows input admittance measurements carried out by the hammer method approximately a year apart. For both measurements the cello was held in the steel rig. The comparison shows very good general agreement, but individual deviations are apparent up to about $5 \mathrm{~dB}$ in amplitude and $5 \mathrm{~Hz}$ shift in modal frequencies. These results set the standard of comparison when looking at the other excitation methods: differences need to be bigger than those seen here before they should be attributed to systematic effects relating to the methods, rather than simply to the limitations on repeatability inherent in tests of this kind.

\section{B. Different driving methods}

A comparison between results measured by the wirebreaking method and bowing method is given in Figure 9. The dashed curve denotes the mean input admittance by the bowing method while the solid one indicates that acquired from the wire-break method. The results show a striking level of similarity between the two admittance curves. It is worth noting that the wire-breaking and bowing methods use exactly the same sensors for both input and output, so differences between the curves here can only be attributed to systematic differences between the forcing methods, or to random effects of nonreproducibility.

In fact, the deviations between the two curves are 


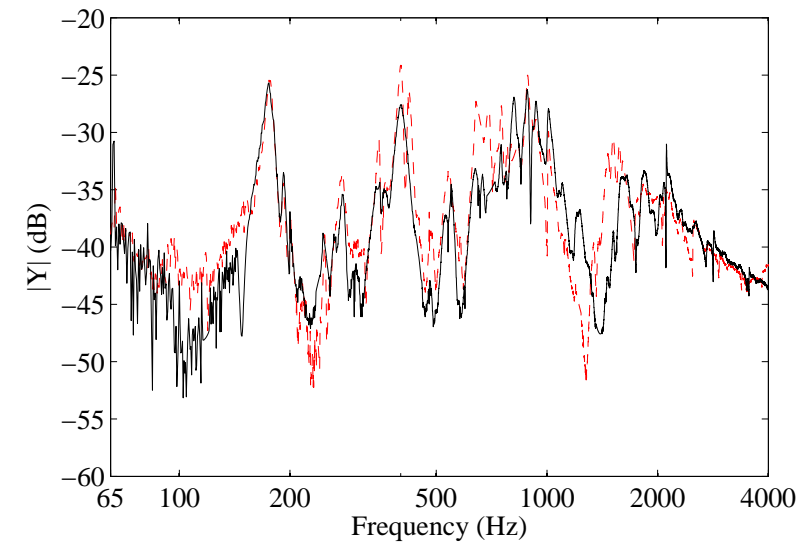

FIG. 8. Input admittances for the tested cello measured on May 2012(dashed line) and March 2013 (solid line).

scarcely more significant than was seen in Figure 8 from nominally identical hammer measurements. At high frequencies, both transfer functions show clear effects of noise pickup, especially the wire-breaking result, but in general these results suggest that there is no major difference between exciting the cello by bowing a string in the normal way, or by applying a controlled external force artificially. In particular, any effects of forces exerted by the vibrating string at other positions or in other directions must be small, because the wire-break method applies force input unambiguously at the string notch and in the bowing direction only.

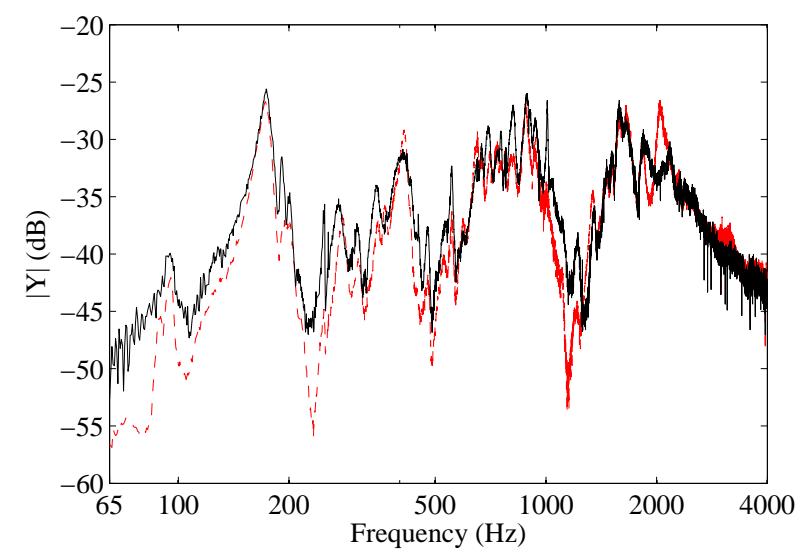

FIG. 9. Input admittances for the tested cello measured by the bowing (dashed line) and wire-breaking (solid line) methods.

Figure 10 shows a comparison of input admittance measured by the hammer method and the bowing method. Differences can be seen, a little greater than those seen in either of the previous comparisons. Of course, the hammer method is expected to be somewhat different from the bowing method because the force is applied in a different place and with less accurate control over the forcing direction. But the similarity between the results in this plot suggests that there is nothing fun- damentally wrong with hammer testing by the familiar method. Given its advantages in simplicity, and crucially the fact that it does not require the force transducer embedded in the instrument bridge, it may be tentatively concluded that the method is accurate enough for measuring vibration behaviour of bowed-string instruments. Similar findings can be observed by comparing the results of the hammer and wire-breaking methods.

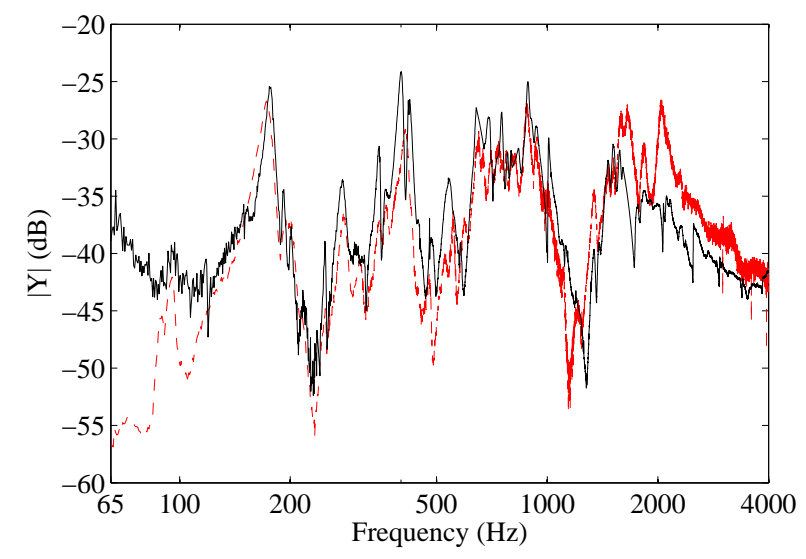

FIG. 10. Input admittances for the tested cello measured by the hammer (solid line) and bowing (dashed line) methods.

\section{Different boundary conditions}

Next, the effect of boundary conditions is examined. The frequency responses using three different holding methods are compared in Figures 11 and 12. The solid curves in both figures indicate the input admittance of the cello held in the steel rig. The dashed curves in Figures 11 and 12 denote the results supported on soft foam and held by a player respectively. To illustrate quantitative modal changes, Table II gives the comparison of the modal properties of the wolf note peak for the three different holding configurations. Small shifts in mode frequency are seen, and holding by the player produces slightly higher damping (lower Q factor).

In Figure 11, the results are consistent in the frequency range of interest. The most obvious difference between these two curves lies in the fact that the absolute level of the mean admittance curve measured in the steel rig is a little higher than the results supported on foam, especially in the vicinity of bridge hill, possibly as a result of slightly higher damping at higher frequencies from the foam supports. Also, the holding method has a small effect on the resonance frequencies. Neither of these observations seems very surprising.

The greater consistency between the results shown in Figure 12 demonstrates the similarity between the steel testing rig and the holding method used by cellists. However, Table II suggests that the steel rig produces a more pronounced frequency shift in the wolf peak than the other two holding methods. 


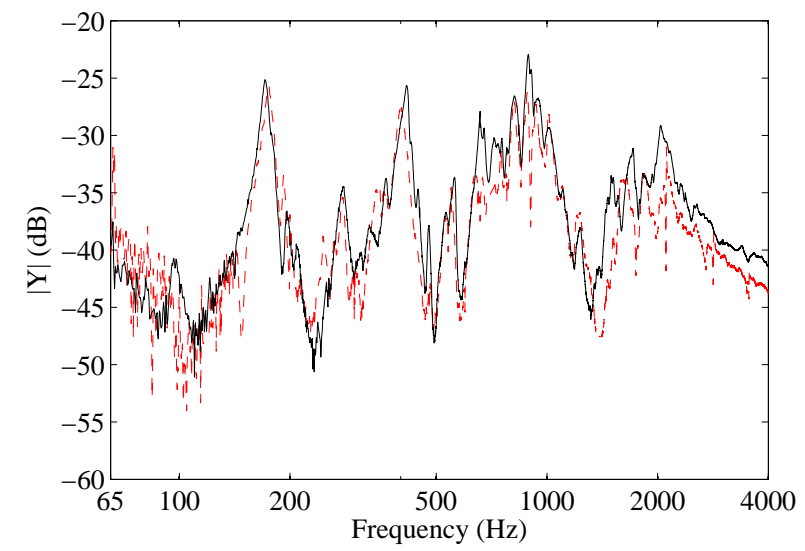

FIG. 11. Input admittances for the tested cello clamped by a steel rig (solid line) and supported by foams (dashed line).

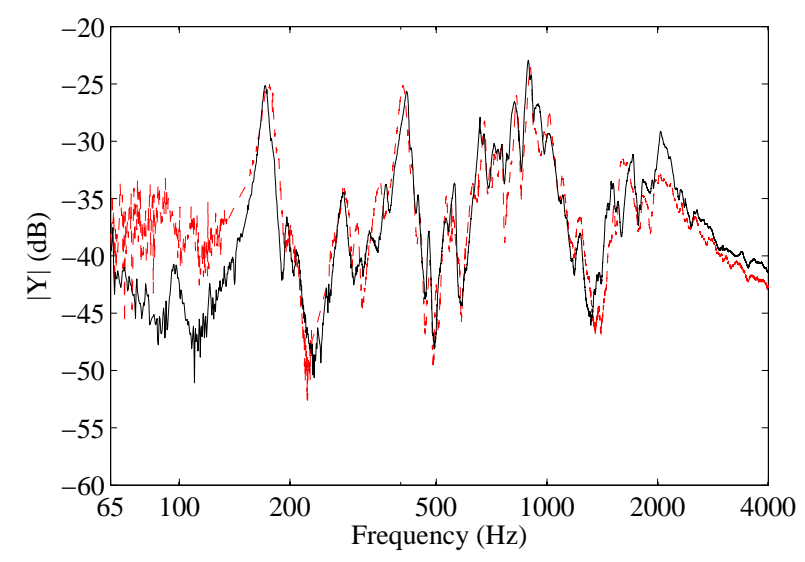

FIG. 12. Input admittances for the tested cello clamped by a steel rig (solid line) and held by a player (dashed line).

TABLE II. Measured resonance frequencies, damping factors and amplitude level in low frequency range.

\begin{tabular}{|c|c|c|c|}
\hline $\begin{array}{c}\text { Different holding } \\
\text { methods }\end{array}$ & $\begin{array}{c}\text { Resonance } \\
\text { frequency } \\
(\mathrm{Hz})\end{array}$ & $\begin{array}{c}\mathrm{Q} \\
\text { factor }\end{array}$ & $\begin{array}{c}\text { Ampli- } \\
\text { tude level } \\
(\mathrm{dB})\end{array}$ \\
\hline Steel rig & 171.7 & 18.1 & -25.1 \\
\hline Foam & 176.5 & 18.5 & -25.6 \\
\hline Player & 175.1 & 13.4 & -25.0 \\
\hline
\end{tabular}

\section{Input admittances of individual strings}

Figures 13 and 14 show a series of measurements using the wire-break method. The two results in Figure 13 are measured transfer functions at the $\mathrm{C}$ string and $\mathrm{G}$ string bridge notches separately, while Figure 14 shows corresponding results at the $\mathrm{D}$ string and A string notches. All the curves show the same set of resonance peaks with different amplitudes, and have no prominent bridge hill because of the mass-loading effects from four pick-ups. Figures 15 and 16 show a corresponding set of separate transfer functions at the four string notches, measured with excitation by bowing. It should be emphasised that all these measurements are strictly transfer admittances: the force is applied at each separate string in the relevant bowing direction, but the response is always measured by the same accelerometer at the bridge corner.

These plots highlight the key differences between the driving methods from the point of view of noise. The wire-break results tend to become noisy and unreliable at high frequencies, as a result of the low energy input from the breaking of the (very thin) wire. The bowing results are better in this regard, although high-frequency noise is still evident. But the most conspicuous noise-related features in the bowing results come at low frequencies, for a very simple reason. The plotted frequency range extends down to $65 \mathrm{~Hz}$, the fundamental of the open $\mathrm{C}$ string. When bowing on the $\mathrm{C}$ string with the octave glissando technique, the full frequency range is covered. However, on the higher strings there is very little energy input below the corresponding fundamental frequency of each string in turn, and that sequence of fundamental frequencies is clearly reflected in the range of low-frequency noise in each measurement. However, even under this noise it can be seen that the general trend of the admittance at low frequencies is still captured because the transients induced by bowing do contribute some energy even at these low frequencies.

From all these results it is clear that, as expected, there are differences between the admittances at the four string notches, but that they are relatively minor. The variations underlying these four transfer functions might come from the slightly different bowing directions and different coupling points on the bridge of each string. The differences between these four results could be used to explore variations in the body response to bowing on the separate strings, and consequent differences in perceived playability. This may be a fruitful avenue for future work, but is not pursued here.

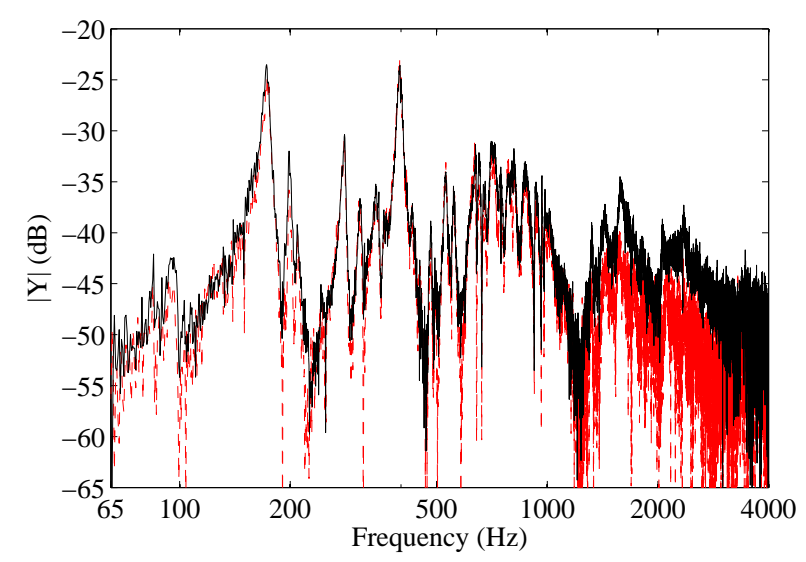

FIG. 13. Input admittances at the bridge notches for the $\mathrm{C}$ string (solid line) and G string (dashed line), measured by the wire-break method.

A typical result obtained by the hammer method is compared with the average of the four transfer functions of the separate strings obtained by the wire-breaking method in Figure 17. These results are striking: they show excellent agreement over the whole frequency range, 


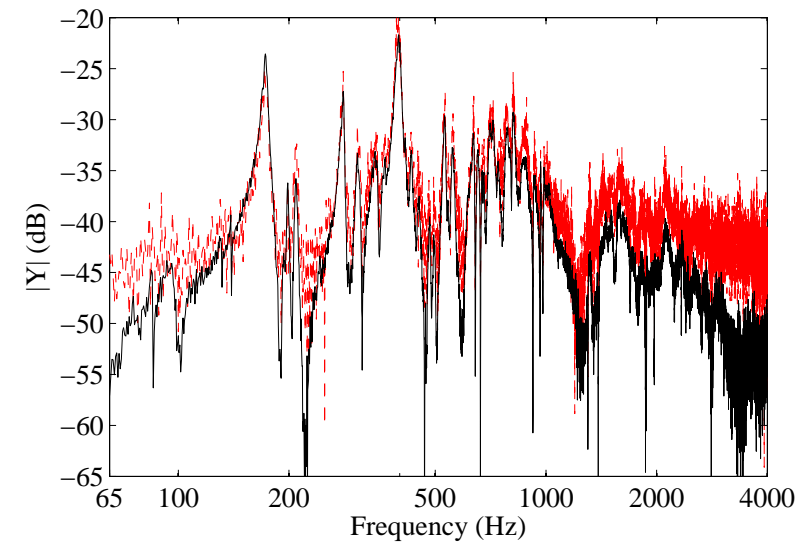

FIG. 14. Input admittances at the bridge notches for the D string (solid line) and A string (dashed line), measured by the wire-break method.

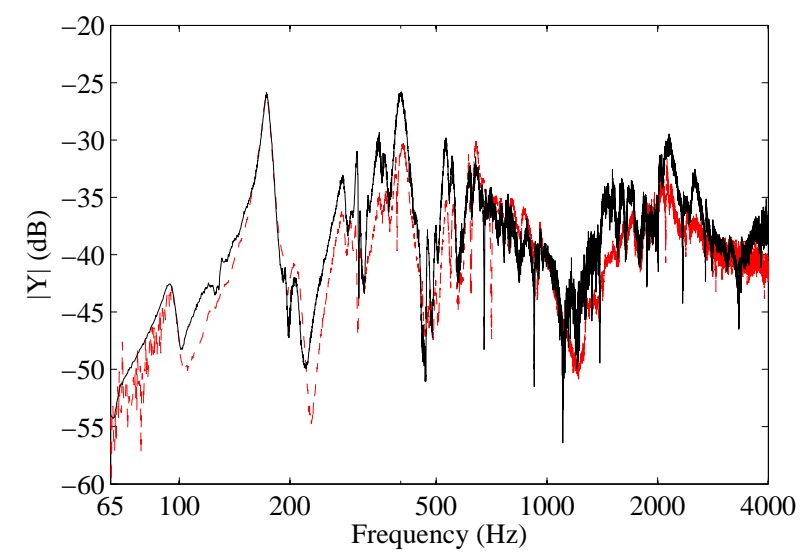

FIG. 15. Input admittances at the bridge notches for the $\mathrm{C}$ string (solid line) and G string (dashed line), measured by the bowing method.

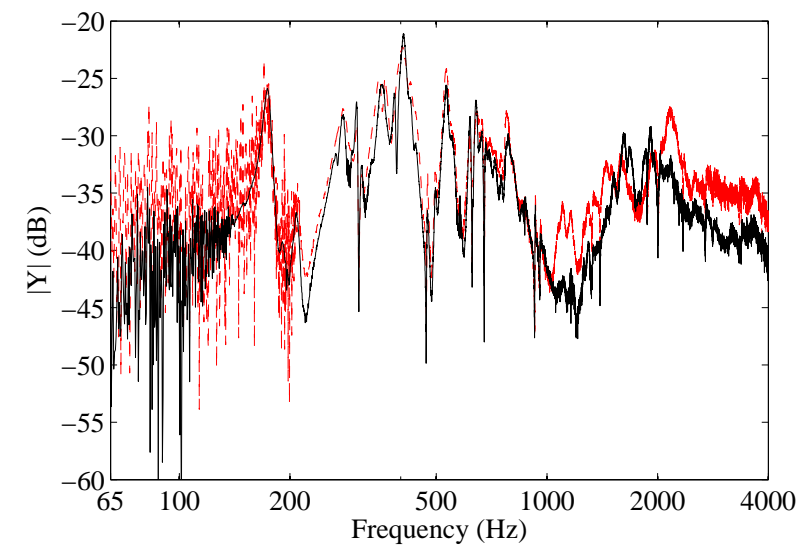

FIG. 16. Input admittances at the bridge notches for the D string (solid line) and A string (dashed line), measured by the bowing method.

which verifies the assumption that the input admittance measured from one bridge corner to another by the ham- mer method could be considered a good first approximation to that of the four strings.

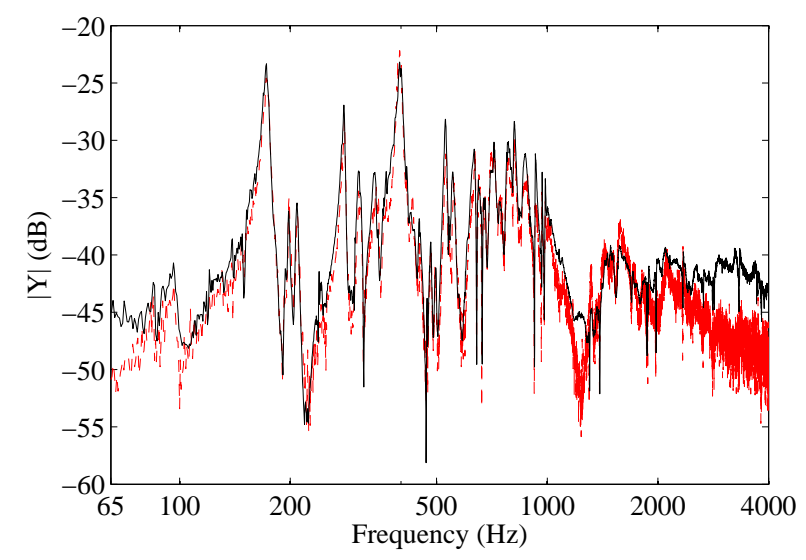

FIG. 17. Input admittances for the tested cello by the hammer method (dashed line) and the average of the admittance at the four separate string notches measured by the wire-break method (solid line).

\section{DISCUSSION AND CONCLUSIONS}

The results of this study give the first direct demonstration of the reliability of the classic hammer method for measuring the vibration behaviour of bowed string instruments, particularly in comparison with excitation by normal bowing. The study relates specifically to the mechanical admittance at the bridge of an instrument. This is a measurement that is particularly important for any study of the interaction between body motion and the behaviour of the bowed string, in other words to issues of playability (Woodhouse, 1993). It should be emphasised that the results shown here only have a somewhat indirect link to the radiated sound from the instrument. Measurements of radiated sound bring in additional complications relating to directional sound radiation and room acoustics. A correspondingly detailed study of radiatedsound transfer functions would require considerable care over those issues.

Comparative results have shown that the measured admittance exhibits only slight differences between different methods of applying force at the cello bridge. Three different holding methods were also studied, including measurements while the instrument was held by a player in the normal way. The results from the three methods agreed almost as closely as repeat measurements by any one method. In this regard the cello is an easier test object than the violin, because the way that a player holds a cello, with the endpin resting on the ground, lends itself fairly readily to copying in a laboratory holding rig.

Using four sets of force sensors embedded in the cello bridge, the input admittance was measured at each of the four separate string notches in the relevant bowing direction. This measurement was possible using two different forcing methods: normal bowing, using an octave 
glissando to fill in the frequency range, or step excitation applied by the breaking of a thin copper wire. The measurements were compared with the commonly-used hammer excitation method, in which impulsive force is applied at one corner of the bridge and response measured at the other corner. This has been shown to give a good approximation to the average of the results for the four separate strings.

It has been shown that the bowing method can be used as a new tool for exploring the characteristics of instruments, but with the drawback that it requires a force sensor on the bridge to measure the input force. The existing sensor is intrusive and not suitable for use on a wide range of instruments, but it is possible that a less intrusive equivalent sensor could be developed, perhaps using Poly vinylidene Difluoride (PVDF) film.

Looking in more detail, these experimental results show some relatively minor differences between the hammer method and bowing method. There are several possible explanations. Firstly, the vibration of the instrument body might not be fully described in terms of linear systems theory. If the instrument body shows some nonlinear behaviour, the principle of superposition utilized in linear systems can no longer be applied to the body vibration and no transfer function can capture the whole behaviour of the body. Secondly, a transversely vibrating string can have two directions of lateral motion, i.e. two polarizations. For this reason, a $2 \times 2$ admittance matrix rather than a single transfer function is required because the input from the string to the body should have two degrees of freedom due to this dual-polarization. Thirdly, there might be axial forces coming from the string, and also both axial and transverse forces at the other end of the string at the fingerboard. There may also be axial force from the other side of the bridge at the tailpiece: even with damped strings, longitudinal vibration in the string may propagate across the bridge.

These additional input forces from bowing are not taken into account in the single-axis transfer functions discussed here. However, the results here show that their net effect on body vibration cannot be large, at least as measured at the bridge, because the results obtained with normal bowing (which includes all these additional forces) were almost indistinguishable from results obtained from wire-break testing at the same string notch (which provides no additional force components).

The last difference between the bowing and hammer impact concerns the direction and location of the hammer force. The hammer cannot in practice be applied exactly at the string notch, so the force input is inevitably a little different. The direction of the force also raises questions: hitting a wooden bridge in the bowing direction for the nearest string means hitting the bridge corner not quite perpendicular to its cut face, and it is hard to be sure precisely what force is in reality applied. One might suspect that variations from this source may be the primary source of differences between different excitation methods reported in the literature. However, the results shown in this paper suggest that none of these factors has such a strong effect as to render hammer measurements seriously in error, provided those measurements are done with care over the forcing and sensing directions.

The remarks about differences between the admittances relevant to the four separate cello strings point towards avenues for further research based on the measurements here. It would be of interest to relate these differences to detailed analysis of the motion of the cello bridge as a function of frequency. This might lead to enhanced understanding of differences of playability between the strings on an instrument, as commonly reported by players.

Moral J.A. and Jansson, E. V. (1982). "Eigenmodes, input admittance and the function of the violin", Acustica 50, 329337.

Cremer, L. (1985). The Physics of the Violin 35-39 (MIT Press Boston, MA).

Woodhouse, J. (1993). "On the playability of violins: Part 2 Minimum bow force and transients", J. Acoust. Soc. Am. 78, 137-153.

Jansson, E. V.and Niewczyk B. K. (1999). "On the acoustics of the violin: bridge or body hill", J. Acoust. Soc. Am. 2(3), 23-27.

Woodhouse, J. (2014). "The acoustics of the violin: a review", Rep. Prog. Phys. In press.

Jansson, E. V. (1997). "Admittance measurements of 25 high quality violins", Acta Acustica united with Acustica 83, 337341.

Harris, N. and Fahy, F. J. (2009). "A comparative study of the hammered bridge response and the bowed string response of the violin", J. Acoust. Soc. Am. 22(1), 211-223.

Marshall, K. D. and Genter, B. (1986). "The musician and the vibrational behaviour of a violin", Catgut Acoust. Soc. Newsletter 45, 28-33.

Fritz, C., Cross, I., Woodhouse, J., and Moore, B. C. J. (2007). "Perceptual thresholds for detecting modifications applied to the acoustical properties of a violin", J. Acoust. Soc. Am. 122, 3640-3650.

Firth, I. M. (1976). "Small mechanical impedance head for use with musical instruments", Acoustica 35, 348-349.

Trott, W. J. (2009). "A comparative study of the hammered bridge response and the bowed string response of the violin", J. Acoust. Soc. Am. 22(1), 211-223.

Jansson, E. V., Bork, I., and Meyer, J. (1986) "Investigations into the acoustical properties of the violin", Acustica 62, 115 .

Jansson, E. V. and Morlin, N. E. (2006). "Laser vibrometry measurements of vibration and sound fields of a bowed violin", Meas. Sci. Technol. 17, 635-644.

Lambourg, C. and Chaigne, A.. (1994). "Measurements and modelling of the admittance matrix at the bridge in guitars", in Proc. Stockholm Music Acoust Conference 2003, (Royal Swedish Academy of Music, Stockholm).

Boutillon, X., Weinreich, G., and Michael, N. R. (1999). "Experimental developments for the measurement of violin bridge admittance", J. Acoust. Soc. Am. 84, S179-S179.

Yoder, L. (1991). "Violin admittance measurements using a one-dimensional mass-loading technique", J. Acoust. Soc. Am. 89, 1878-1878.

Woodhouse, J. and Courtney, P. E.. (2003). "The admittance matrix of a cello", in Proc. Stockholm Music Acoust Conference 2003, (Royal Swedish Academy of Music, Stockholm). Ewins, D. J. (1986). Modal testing: theory, practice, and application 140-141 (Letchworth: Research studies press). 